C-A/AP/\#409 October 2010

\title{
RHIC Electron Lens Beam Transport System Design Considerations
}

X. Gu, A. Pikin, M. Okamura, W. Fischer, Y. Luo, R. Gupta, J. Hock, A. Jain, D. Raparia

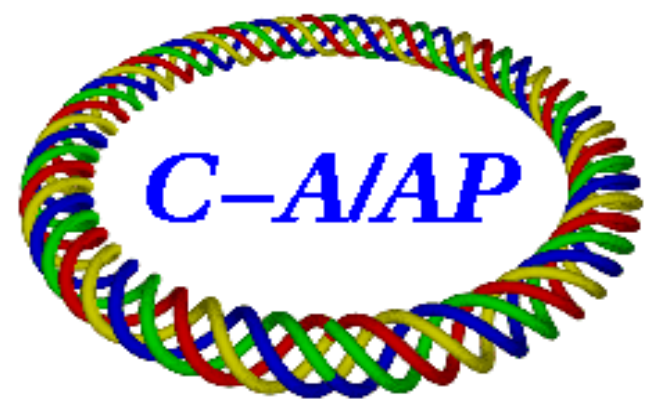

\section{Collider-Accelerator Department Brookhaven National Laboratory Upton, NY 11973}

Notice: This document has been authorized by employees of Brookhaven Science Associates, LLC under Contract No. DE-AC02-98CH10886 with the U.S. Department of Energy. The United States Government retains a nonexclusive, paid-up, irrevocable, world-wide license to publish or reproduce the published form of this document, or allow others to do so, for United States Government purposes. 


\title{
RHIC Electron Lens Beam Transport System Design Considerations
}

\author{
X. Gu, A. Pikin, M. Okamura, W. Fischer, Y. Luo, R. Gupta, J. Hock, A. Jain, D. Raparia
}

Brookhaven National Laboratory, Upton, NY 11973

\begin{abstract}
August 24, 2010
To apply head-on beam-beam compensation for RHIC, two electron lenses are designed and will be installed at IP10. Electron beam transport system is one of important subsystem, which is used to transport electron beam from electron gun side to collector side. This system should be able to change beam size inside superconducting magnet and control beam position with $5 \mathrm{~mm}$ in horizontal and vertical plane.
\end{abstract}

Some other design considerations for this beam transport system are also reported in this paper.

\section{Introduction}

The head-on beam-beam effect is one of important nonlinear source in storage ring and linear colliders, which have limited the luminosity improvement of many colliders, such as SppS, Tevatron and RHIC. In order to enhance the performance of colliders, beam-beam effects can be compensated with direct space charge compensation, indirect space charge compensation or betatron phase cancelation scheme.

Like other colliders, indirect space charge compensation scheme (Electron Lens) was also proposed for Relativistic Heavy lon Collider (RHIC) beam-beam compensation at Brookhaven National Laboratory. The two similar electron lenses are located in IR10 between the DX magnets. One RHIC electron lens consists of one DC electron gun, one superconducting magnet, one electron collector and beam transport system.

First of all, to design electron lens beam transport system, the most important thing is to transport electron beam from gun side to collector side, control electron beam trajectories and make them follow the center line of superconducting main magnet (SM).

The gun and collector have almost identical solenoid. Six conventional magnets are used for this purpose and placed symmetry to center of SM. Fig. 1 is the layout of one electron lens, which has gun 
side, SM and collector side. Each side of one electron lens has three magnets; they are GS1, GS2 and GSB on left side, and CS1, CS2 and CSB on right side.

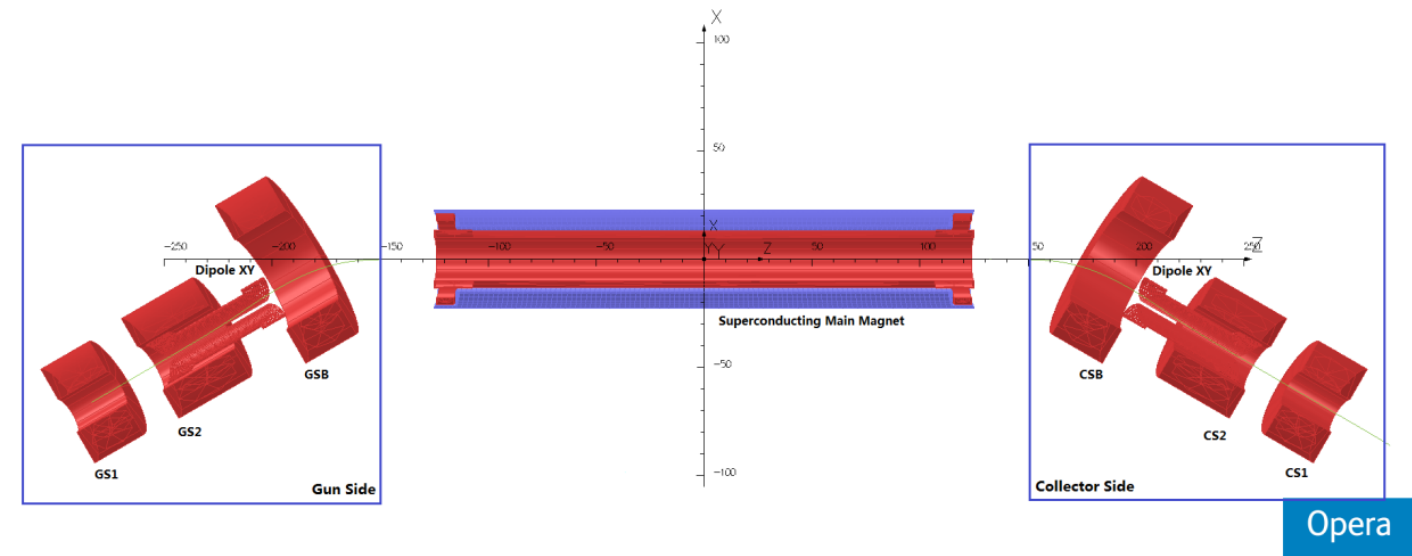

Fig. 1 Layout of E lens

According to beam physics acquirements, the electron beam size should be changed to match the proton size inside SM, which is their interaction area. The electron beam size in main superconducting magnet is calculated by:

$$
\sigma_{\mathrm{e}}=\sigma_{\text {gun }} \cdot \sqrt{\frac{\mathrm{B}_{\text {gun }}}{\mathrm{B}_{\text {main }}}}
$$

Where $\sigma_{\text {gun }}$ is the beam size from electron gun cathode, $B_{\text {gun }}$ and $B_{\text {main }}$ are magnetic field in electron gun and main superconducting magnet respectively.

So secondly, this beam transport system should have the capability to change the magnetic field ratio between SM and GS1. GS1 can change it field from $0.2 \mathrm{~T}$ to $0.8 \mathrm{~T}$ and magnetic field of SM can be changed from $1 \mathrm{~T}$ to $6 \mathrm{~T}$. This will give us almost 5 times beam size change from its minimum value $\sigma_{\text {gun }} \cdot \sqrt{\frac{1}{30}}$ to its maximum value $\sigma_{\text {gun }} \cdot \sqrt{\frac{4}{5}}$. And when changing GS1 field or changing beam size, GS1 field will not affect electron beam trajectories. Beam size change and trajectories change are independently.

Thirdly, in addition to transporting electron beam and controlling beam size inside SM, electron transport system also should have the magnetic field that large enough to suppress unwanted space charge effects, and should be rigid enough that electron beam can't be disturbed by or disturb other electromagnetic field.

For our electron lens beam transport system, the fourth important thing is to steer beam position inside SM magnet with $5 \mathrm{~mm}$ change in horizontal and vertical plane. To satisfy this requirement, two dipole magnets (Dipole $X$ and Dipole $Y$ ) are designed for each side of two lenses. 
At last, during electron lens design period, running cost also should be taken for considerations. How to reduce power consumption used in transport system is another important thing. The power consumption for both electron lenses should be limited to $500 \mathrm{~kW}$ in order to avoid upgrades to the electrical and water cooling system in IR10.

After that, some other design considerations, such as two electron lens simulations, power stability, iron thickness (for superconducting main magnet) and realistic solenoid simulations are also discussed in this note.

\section{Electron Beam Center Trajectory Control}

With electron lens default operation configuration, electron beam comes out from electron gun first, and then goes through GS1, GS2 and GSB. After that, it starts to enter the SM magnet, passes SM along the center line of this magnet. Then, it is transported from CSB, CS2 and CS1. Finally, it is dumped into a collector. This procedure is also shown as the green line in Fig. 1, which starts from gun side to collector side.

With default operation configuration, dipole magnet should be shut down, and the magnetic field along this line should be greater than $0.3 \mathrm{~T}$.

There are several parameters that can be used for beam trajectories control, such as the strength of GSB and SM magnetic field, the angle between GSB and $z$ axis and the distance between GSB and SM. Table 1 lists all parameters which can be used for trajectory control, and shows beam trajectories behaviors when these parameters are applied.

Table1 the Parameters for Beam Center Trajectory Control

\begin{tabular}{rrr}
\hline \hline Parameters & Beam Position Move Up & Beam Position Move Down \\
\hline GSB current & Increase & Decrease \\
GSB angle & Increase & Decrease \\
GSB position & Shift up & Shift down \\
GS1 and GS2 angle & Decrease & Increase \\
GS1 and GS2 position & shift to left & shift to right \\
GSB, GS1 and GS2 position & Shift to left & Shift to right \\
GSB Local Shift & Shift up & Shift down \\
GS1 and GS2 current & No change \\
\hline \hline
\end{tabular}

Table 2 is our electron lens beam transport system design specifications. The first part in this table includes the position and angle of GS1, GS2 and GSB. The second part of this table is the conductor parameters and the geometry of these magnets. The third part that listed in this table is the power consumption, temperature increase and magnetic field which are caused by these solenoids, and they are given with two different cases, normal optimization case and normal plus $40 \%$ current case. 
Table 2 E-lens Beam Transport system Design Specifications

\begin{tabular}{|c|c|c|c|c|c|c|}
\hline & \multicolumn{6}{|c|}{$\begin{array}{l}\text { E-lens Beam Transport System Design } \\
\text { Specification }\end{array}$} \\
\hline & & GS1 & GS2 & GSB & GSX & GSY \\
\hline \multicolumn{7}{|c|}{ Position and Angle } \\
\hline Global Position & L_*_GCS (mm) & -1690 & -1690 & -1850 & -1690 & -1690 \\
\hline Local Position & $\mathrm{L}_{-}^{*}{ }_{-} \mathrm{LCS}(\mathrm{mm})$ & 1320 & 820 & 100 & 660 & 660 \\
\hline Angle & Theta (degree) & 30 & 30 & 30 & 30 & 30 \\
\hline \multicolumn{7}{|c|}{ Solenoid Parameters } \\
\hline \multirow{3}{*}{ Conductor } & h_cond (mm) & 14 & 14 & 14 & 6.35 & 6.35 \\
\hline & ID_water(mm) & 9 & 9 & 9 & 4.75 & 4.75 \\
\hline & b_insul (mm) & 0.3 & 0.3 & 0.3 & 0.65 & 0.65 \\
\hline \multirow{8}{*}{ Size } & $\mathrm{ID}(\mathrm{mm})$ & 173.5 & 234 & 480 & 194 & 210 \\
\hline & $\mathrm{OD}(\mathrm{mm})$ & 553.1 & 526 & 859.6 & 208 & 224 \\
\hline & Length(mm) & 262.8 & 379.6 & 262.8 & 500 & 500 \\
\hline & N_Layer & 13 & 10 & 13 & 12 & 12 \\
\hline & N_pan & 9 & 13 & 9 & & \\
\hline & Inductance (Henry) & 0.02 & 0.02 & 0.04 & & \\
\hline & Resistance (ohm) & 0.04 & 0.05 & 0.08 & 0.02 & 0.02 \\
\hline & \multicolumn{6}{|c|}{ Optimization } \\
\hline \multirow[b]{2}{*}{ Power } & Power (kW) & 58.3 & 25.6 & 45 & 1.4 & 1.7 \\
\hline & Current(A) & 1188 & 731 & 769 & 258 & 271 \\
\hline & Temp_Delta $\left({ }^{\circ} \mathrm{C}\right)$ & 13.4 & 3.6 & 14.2 & 5.9 & 6.9 \\
\hline Water & Pressure_Drop(Bar) & 1.5 & 1.5 & 1.5 & 1.5 & 1.5 \\
\hline \multirow[t]{2}{*}{ Field } & (Gauss) & 8000 & 4468 & 3202 & $190 *$ & $190^{*}$ \\
\hline & \multicolumn{6}{|c|}{ Plus $40 \%$ Current } \\
\hline \multirow[b]{2}{*}{ Power } & Power (kW) & 114 & 50 & 88 & 2.9 & 3.4 \\
\hline & Current(A) & 1663 & 1023 & 1077 & 361 & 383 \\
\hline \multirow[b]{2}{*}{ Water } & Temp_Delta $\left({ }^{\circ} \mathrm{C}\right)$ & 26 & 7 & 8 & 12 & 14 \\
\hline & Pressure_Drop(Bar) & 1.5 & 1.5 & 1.5 & 1.5 & 1.5 \\
\hline Field & (Gauss) & 11200 & 6256 & 4482 & $270^{\wedge}$ & $270^{\wedge}$ \\
\hline
\end{tabular}

- $\quad *$ is the dipole magnetic field for $5 \mathrm{~mm}$ beam shift.

- $\wedge$ is the dipole magnetic field for $7 \mathrm{~mm}$ beam shift. 
Because CS1, CS2 and CSB have the same configuration as GS1, GS2 and GSB respectively, they are not listed in this table.

Fig. 2 shows the position definitions that are listed in table 2. GS1 and GS2 have the same global position, which is L_GS12_GCS. GSB has its own global position L_GSB_GCS. But all of them have the same 30 degree angle. And they have their different local positions. From the global position and along 30 degree line, they are moved with the distance L_GS1_LCS, L_GS2_LCS and L_GSB_LCS.

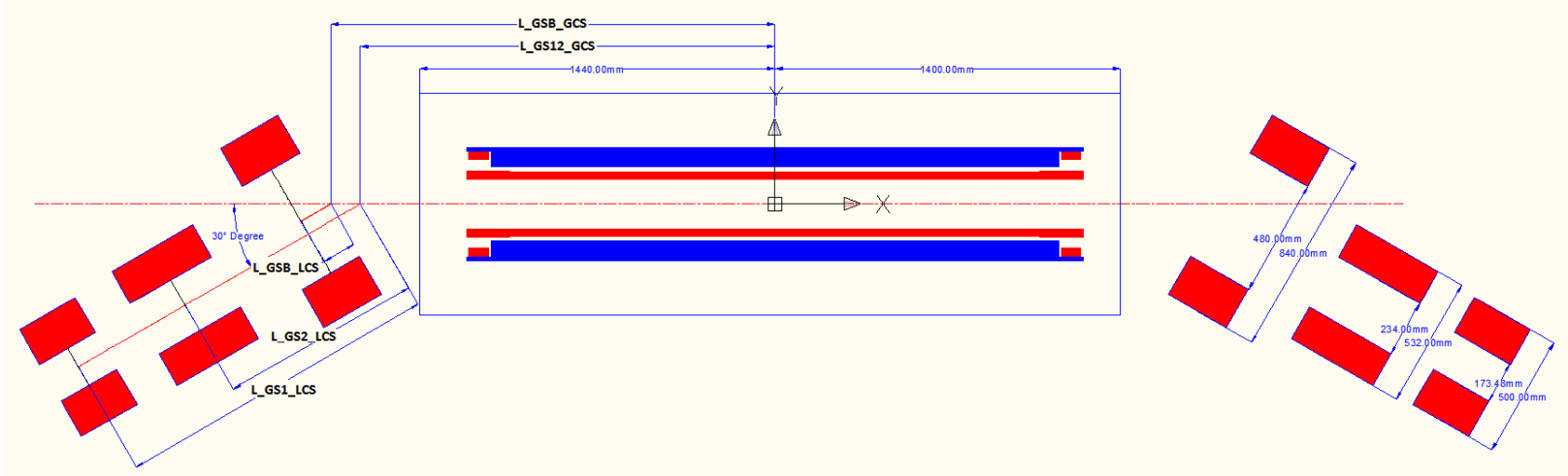

Fig. 2 Position Definitions in Table 2

At last, when electron beam passes this beam transport system, with the parameters listed in table 2, it can go through SM magnet along its center line. Meanwhile, the magnetic field along the center line (the green line in Fig. 1) was also plot as Fig. 3.

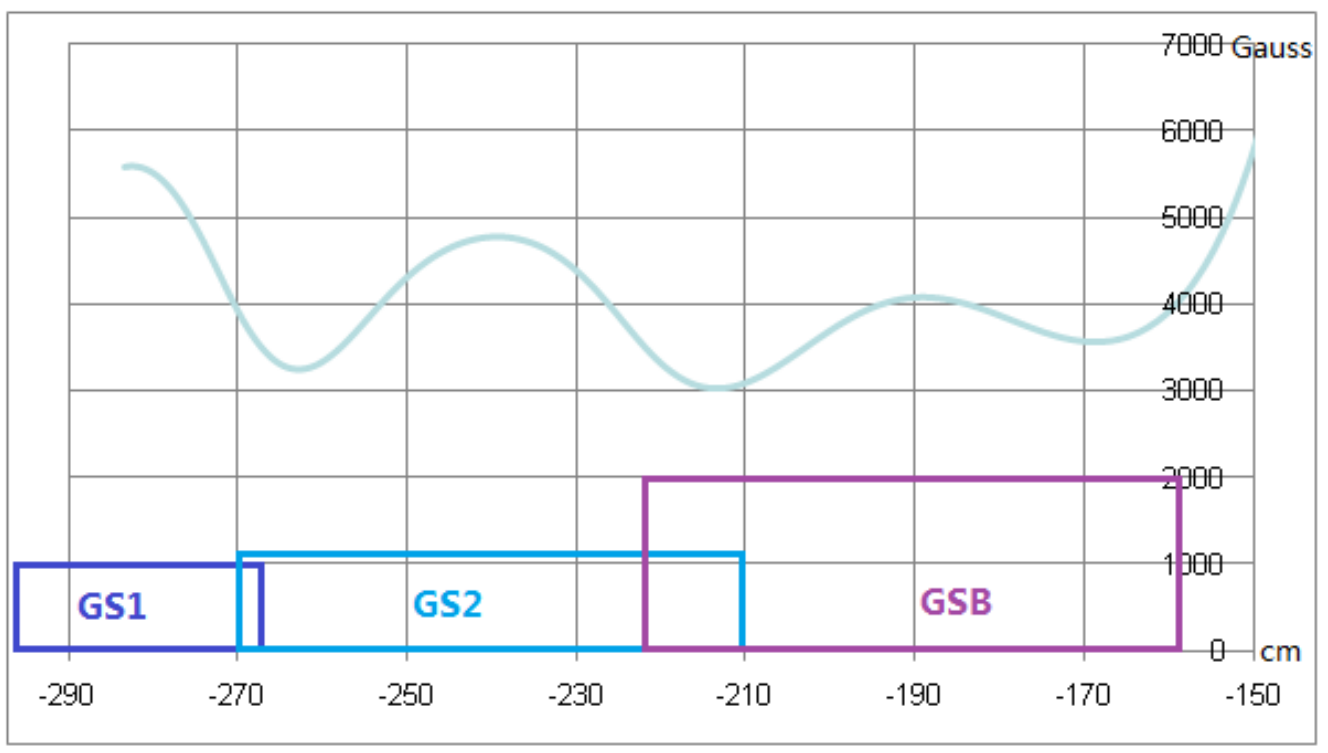

Fig. 3 Magnetic field distribution along center trajectory line 
From Fig. 3, we can find that all positions that electron beam passes, its magnetic field is greater than $0.3 \mathrm{~T}$.

Fig. 4 is the magnetic field map distribution around gun side, which shows the area that the amplitude of magnetic field is greater than $0.3 \mathrm{~T}$.

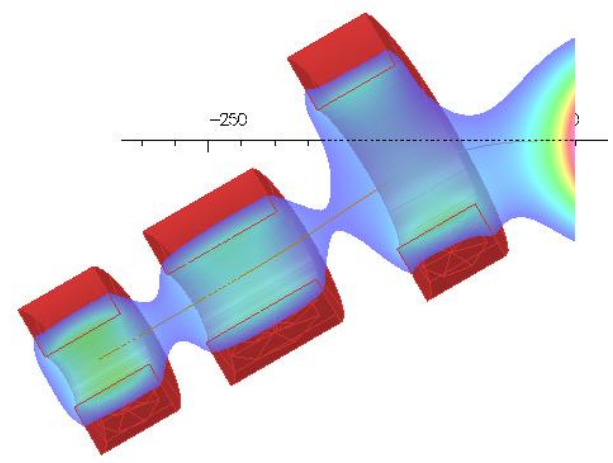

Fig. 4 Magnetic field distribution around gun side

\section{Dipole Magnet Design for Beam Off-set}

For electron lens, electron beam should head on collide with proton beam at IP 6 or IP 8. So, it is very important to align electron beam with proton beam. For this purpose, to control electron is easier than to control proton. Because two proton beams share one beam pipe at IP 10 with $10 \mathrm{~mm}$ vertical distance between then, and we want the electron beam has the capability of shifting $5 \mathrm{~mm}$ around the center line in horizontal and vertical plane. To satisfy this requirement, two dipole magnets (Dipole $\mathrm{X}$ and Dipole Y) are designed for each side of two lenses, and are placed inside of GS2 and CS2.

Their parameters are also listed in Table 2. Fig. 5 is the geometry of one dipole X, and Fig. 6 is its magnetic field distribution. Dipole $\mathrm{Y}$ has almost same geometry and field distribution like dipole $\mathrm{X}$. 


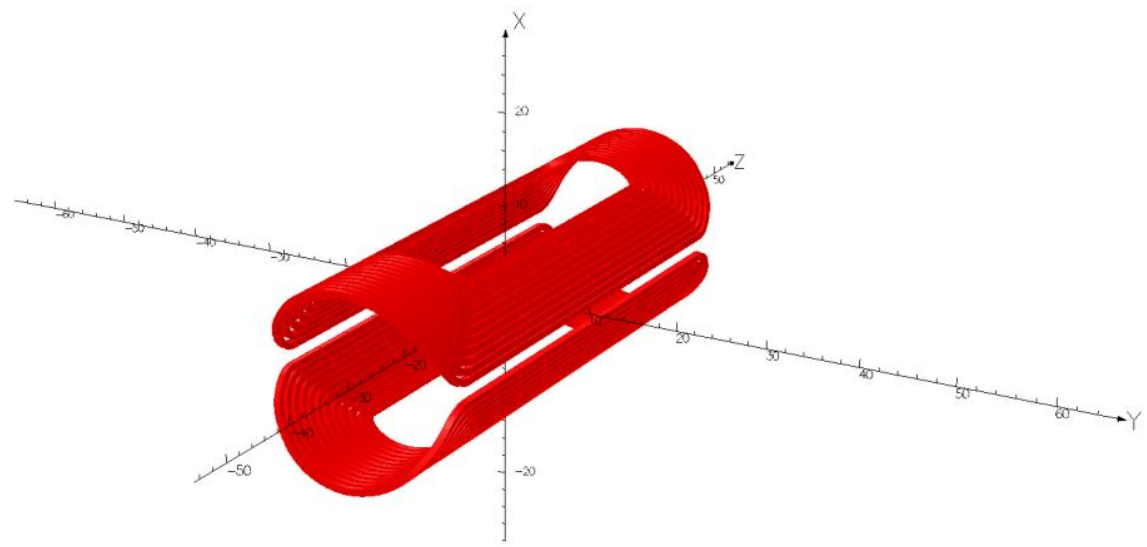

Fig. 5 Geometry of Dipole X

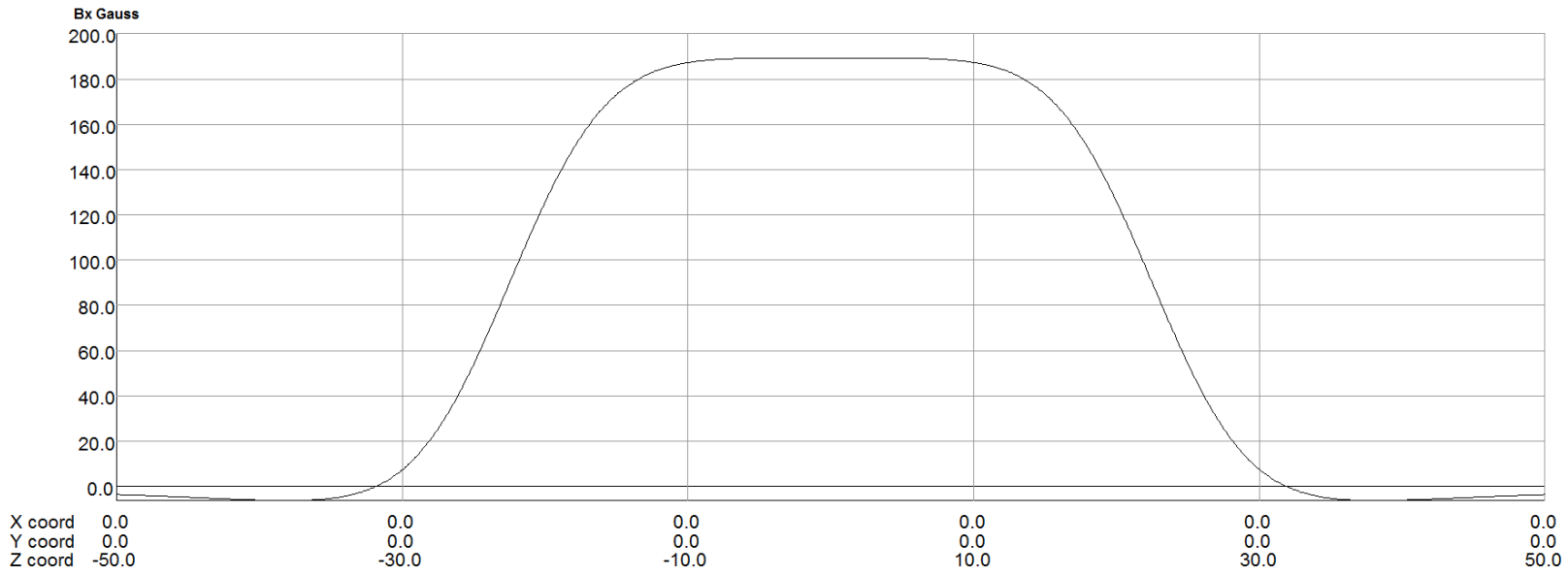

Fig. 6 the Magnetic Field Distribution (Bx) Produced by Dipole X

With this dipole field, the beam trajectories inside of SM magnet can be moved about $5 \mathrm{~mm}$.

Fig. 7 is the beam trajectories envelope after using dipole magnet with the center beam trajectory $5 \mathrm{~mm}$ shift in horizontal direction. In Fig. 7, when the upper line was plot, the electron comes from upper side of cathode and beam was shifted up $5 \mathrm{~mm}$. The lower line was plot with the electron produced by lower side of cathode and with beam $5 \mathrm{~mm}$ shift down.

According to Fig. 7, we can optimize the tube inner diameter so that the electron beam will not touch its inner side. Tube inner size should also be careful design by technician. 


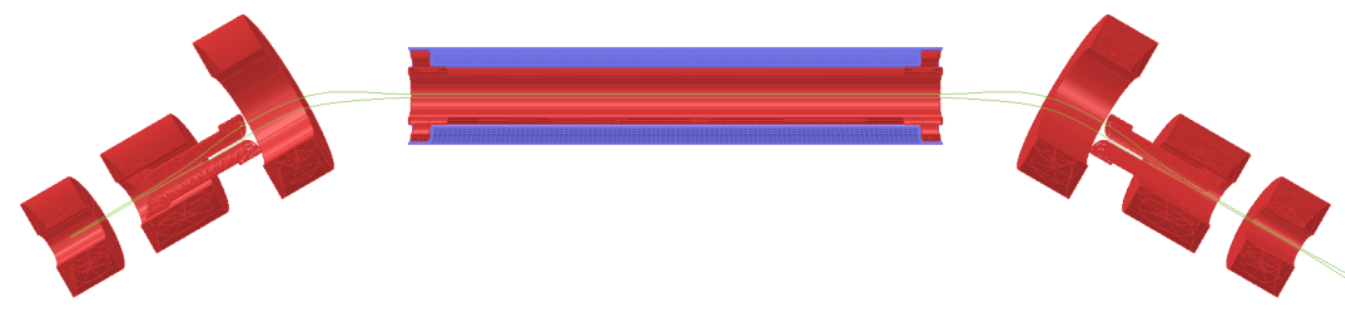

Opera

Fig. 7 Horizontal Beam Trajectory Envelope with 5mm Shift Up and Down)

\section{Power Consumption Optimization}

For power consumption, we tried several approaches. Firstly, we can optimize the conductor parameters.

Table 3 lists some conductors and their power consumptions, currents and space factor. Space factor equals to the conductor intersection area divided by total intersection area. All these conductors are the conductor with square outside and round hole inside. $H_{-}$Cond is the out size and D_water is the inner diameter of water cooling hole.

Table 3 Conductor and Power Consumption

\begin{tabular}{rrrrrr}
\hline \hline Conductor & H_Cond & D_Water & P (kW) & Current (A) & Space Factor $\lambda$ \\
\hline 1 & 9.7 & 7.9 & 77.88 & 418 & 0.425 \\
2 & 11 & 8.8 & 77.58 & 557 & 0.447 \\
3 & 6 & 4.5 & 72.94 & 175 & 0.461 \\
4 & 6.35 & 4.75 & 73.6 & 198 & 0.468 \\
5 & 10 & 7.5 & 71.58 & 480 & 0.497 \\
6 & 7 & 5 & 68.27 & 240 & 0.509 \\
7 & 8 & 5.5 & 64.35 & 311 & 0.544 \\
8 & 13 & 9 & 65.78 & 837 & 0.569 \\
9 & 9.52 & 6.35 & 59.26 & 418 & 0.576 \\
10 & 14 & 9 & 54.5 & 846 & 0.62 \\
\hline \hline
\end{tabular}

Fig. 8 is the power consumptions with different conductors that listed in table 3 . Its vertical unit is Kilowatt. At last, the conductor with number 10 is used for our solenoids magnet design, and the conductor 4 is used for dipole magnet design. 


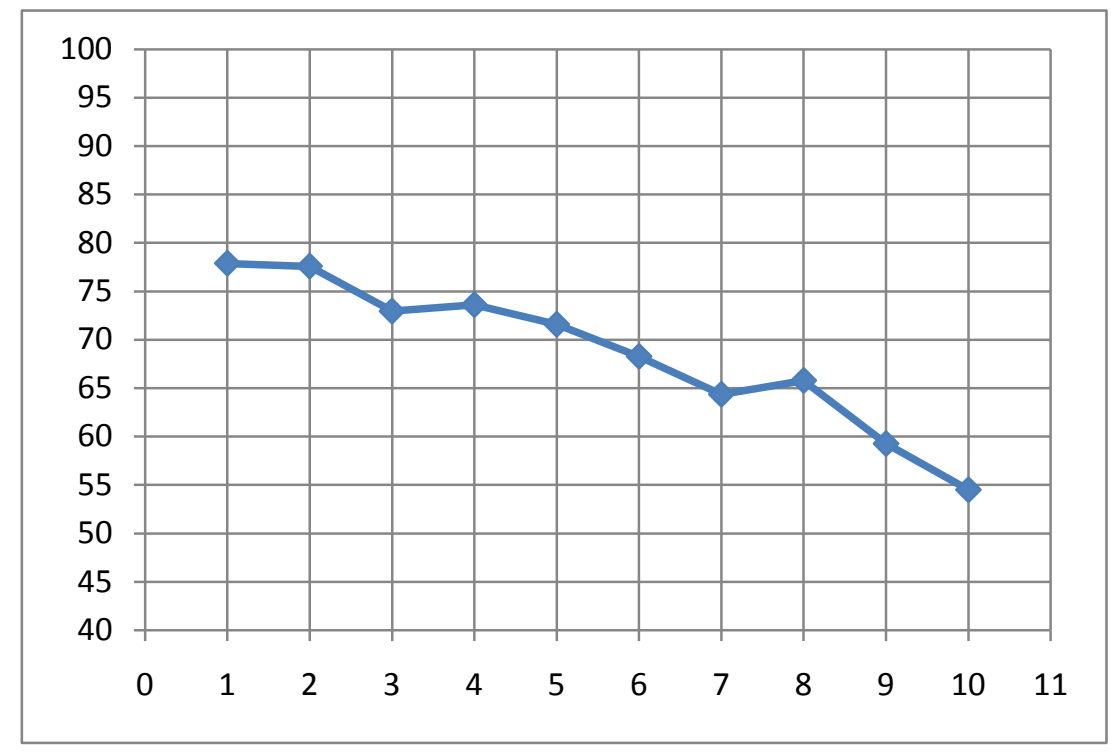

Fig. 8 Power Consumption with Different Conductor

With $0.8 \mathrm{~T}$ in GS1 and $0.4 \mathrm{~T}$ in CS1, the total power consumption is about $430 \mathrm{~kW}$ for two electron lenses.

The second way to reduce power cost is to use different operating configuration. For our default operating model, we tune the current of GSB to control beam trajectories. Dipole magnets are turned off at this moment. After finishing electron lens commissioning, if it is possible to decrease the minimum magnetic field, from $0.3 \mathrm{~T}$ to $0.15 \mathrm{~T}$ for example, maybe we can reduce the GSB current while increase dipole magnet $X$ current for beam position control.

The case $C$ in the Table 4 is one example (not the realistic one) which operated with dipole magnet $\mathrm{X}$. Compared with case $\mathrm{A}$ (default operating model) and case $\mathrm{C}$, we can reduce power consumption for $34 \mathrm{~kW}$.

Table 4 Power Consumption for Different Operating Model

\begin{tabular}{rrrrrr}
\hline \hline & $\begin{array}{r}\text { Dipole X Current } \\
\text { Density(A/cm^2) }\end{array}$ & $\begin{array}{r}\text { GSB Current } \\
\text { Density(A/cm²) }\end{array}$ & $\begin{array}{r}\text { Beam } \\
\text { Position(cm) }\end{array}$ & $\begin{array}{r}\text { Dipole X } \\
\text { Power(kW) }\end{array}$ & GSB Power(kW) \\
\hline A & 0 & 365 & $\sim 0$ & 0 & $\sim 45$ \\
B & 0 & 180 & $\sim-0.48$ & 0 & $\sim 11$ \\
C & 350 & 180 & $\sim-0.02$ & 0.3 & $\sim 11$ \\
\hline \hline
\end{tabular}

Thirdly, we also did some power consumption optimizations for magnets themselves. Fig. 9 is the power optimization for GS1 solenoid. The red curve is the power consumption change with different number of pancakes while number of layer is 13 . The blue curve is the power change with layer number, while keep pancake number same as 9. 


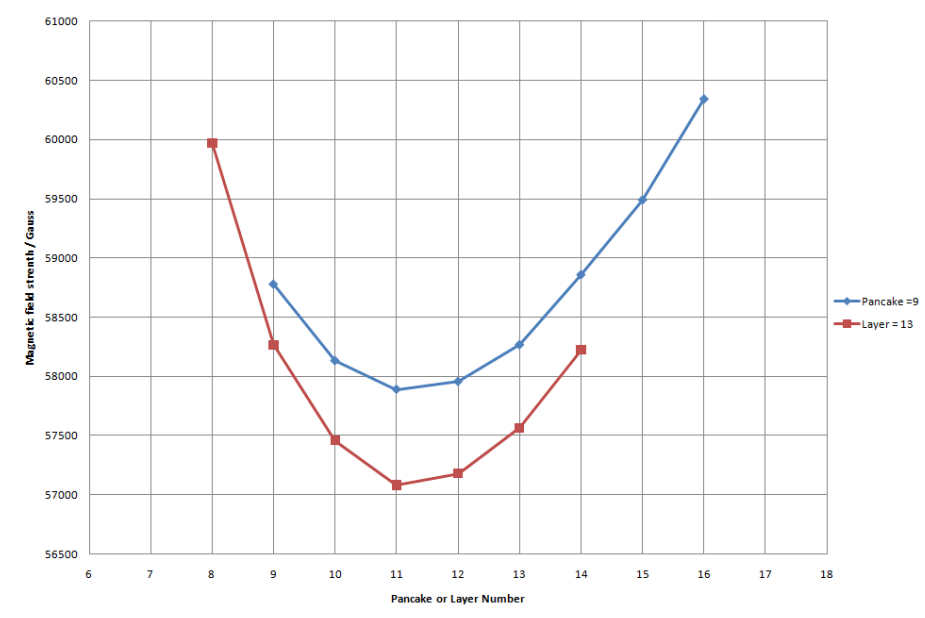

Fig. 9Power Consumption with Different Conductor

From Fig. 9, solenoid GS1 with 11 pancakes and 13 layers has the minimum power consumption $57.08 \mathrm{~kW}$, that is slightly lower than present parameter (9 pancake and 13 layers) $58.26 \mathrm{~kW}$.

\section{Two Electron Lenses Simulations}

For RHIC head-on beam compensation system, it has two electron lenses. Fig. 10 is the layout of two symmetrical electron lenses system.

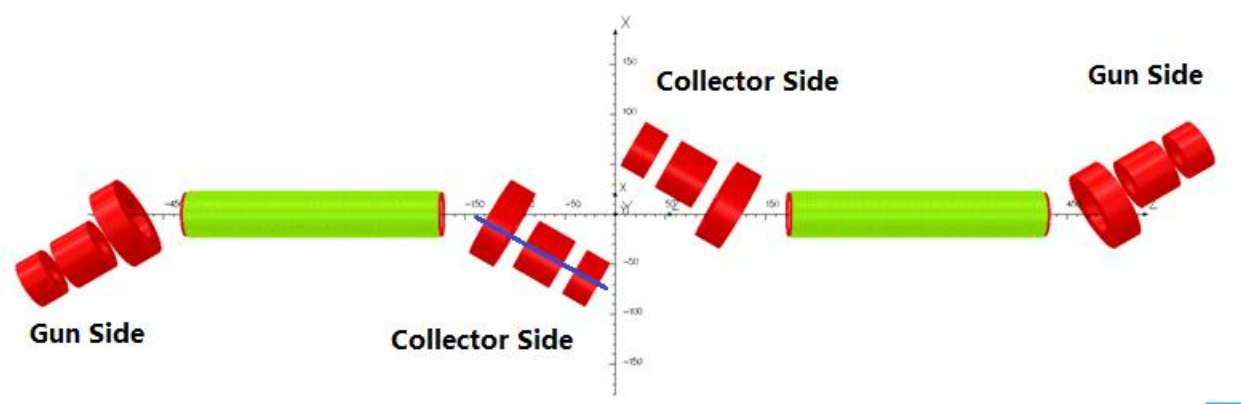

Fig. 10 Layout of Two Electron Lenses

For this two lenses system, the distance between them is another important thing. If we put them too close, their magnetic field on their collector side will affect each other, and electron beam trajectories maybe change too much. In term of magnetic field distort and beam trajectories change, $500 \mathrm{~cm}$ and $600 \mathrm{~cm}$ distances between these two electron lenses were investigated when we carried out our simulations.

Fig. 11 is their magnetic fields which were plot along the blue line in Fig. 10. With right side electron lens power off and power on, the magnetic field change 77 Gauss and 37 Gauss for $500 \mathrm{~cm}$ and $600 \mathrm{~cm}$ distance respectively. 

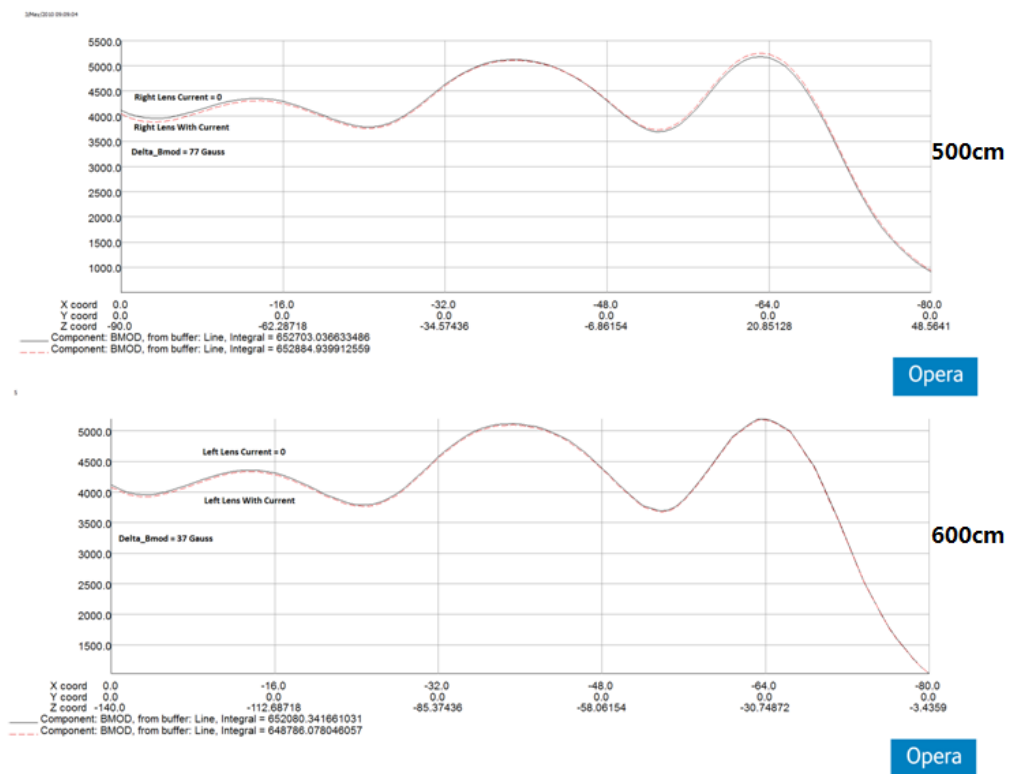

Fig. 11 Magnetic Field change for Two Electron Lens Configurations

Because magnetic field changed, the beam trajectories that located at the center point between CS1 and CS2 also changed. Fig. 12 is the electron beam trajectories change, which is about $48 \mathrm{~mm}$ and $12 \mathrm{~mm}$ for $500 \mathrm{~cm}$ and $600 \mathrm{~cm}$ distance respectively.
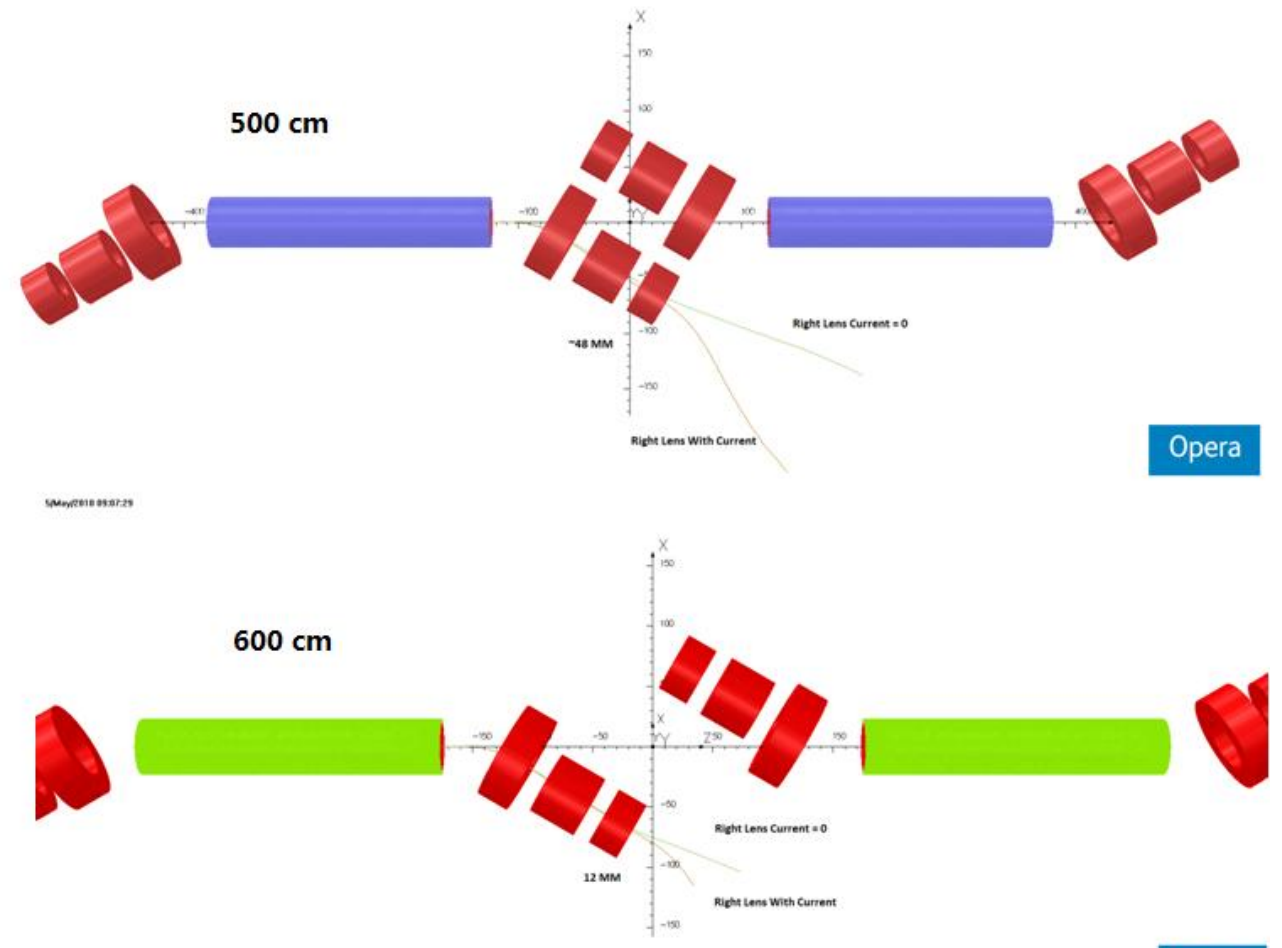
Fig. 12 Magnetic Field change for Two Electron Lens Configurations

During beam transport system design period, magnetic force calculation is another essential problem. Table 5 is the force calculation for left electron lens with $500 \mathrm{~cm}$ and $600 \mathrm{~cm}$ distance between the two lenses.

Table 5 Force Calculation with Different Lens Distance

\begin{tabular}{r|rrr|rrr}
\hline \hline \multirow{2}{*}{ Magnet } & \multicolumn{3}{|c|}{500 CM } & \multicolumn{3}{c}{ 600 CM } \\
\cline { 2 - 6 } & $\mathrm{X}(\mathrm{kN})$ & $\mathrm{Y}(\mathrm{kN})$ & $\mathrm{Z}(\mathrm{kN})$ & $\mathrm{X}(\mathrm{kN})$ & $\mathrm{Y}(\mathrm{kN})$ & $\mathrm{Z}(\mathrm{kN})$ \\
\hline $\mathrm{GSB}$ & -2.4 & 0 & 6.7 & -2.5 & 0 & 6.9 \\
$\mathrm{GS} 2$ & 2.0 & 0 & 2.2 & 2.0 & 0 & 2.1 \\
$\mathrm{GS} 1$ & 1.9 & 0 & 3.5 & 1.9 & 0 & 3.3 \\
\hline $\mathrm{CSB}$ & -2.8 & 0 & -6.8 & -2.7 & 0 & -6.9 \\
CS2 & 1.7 & 0 & -2.0 & 1.9 & 0 & -2.1 \\
CS1 & 2.2 & 0 & -3.0 & 1.8 & 0 & -3.3 \\
\hline \hline
\end{tabular}

\section{Current Stability Calculation for Power Supply}

To estimate the power stability, we change GSB current (or current density) and record the beam position (at the center of SM magnet) change, then plot GSB current as the function of Beam position in Fig. 13 and fit this plot with linear function.

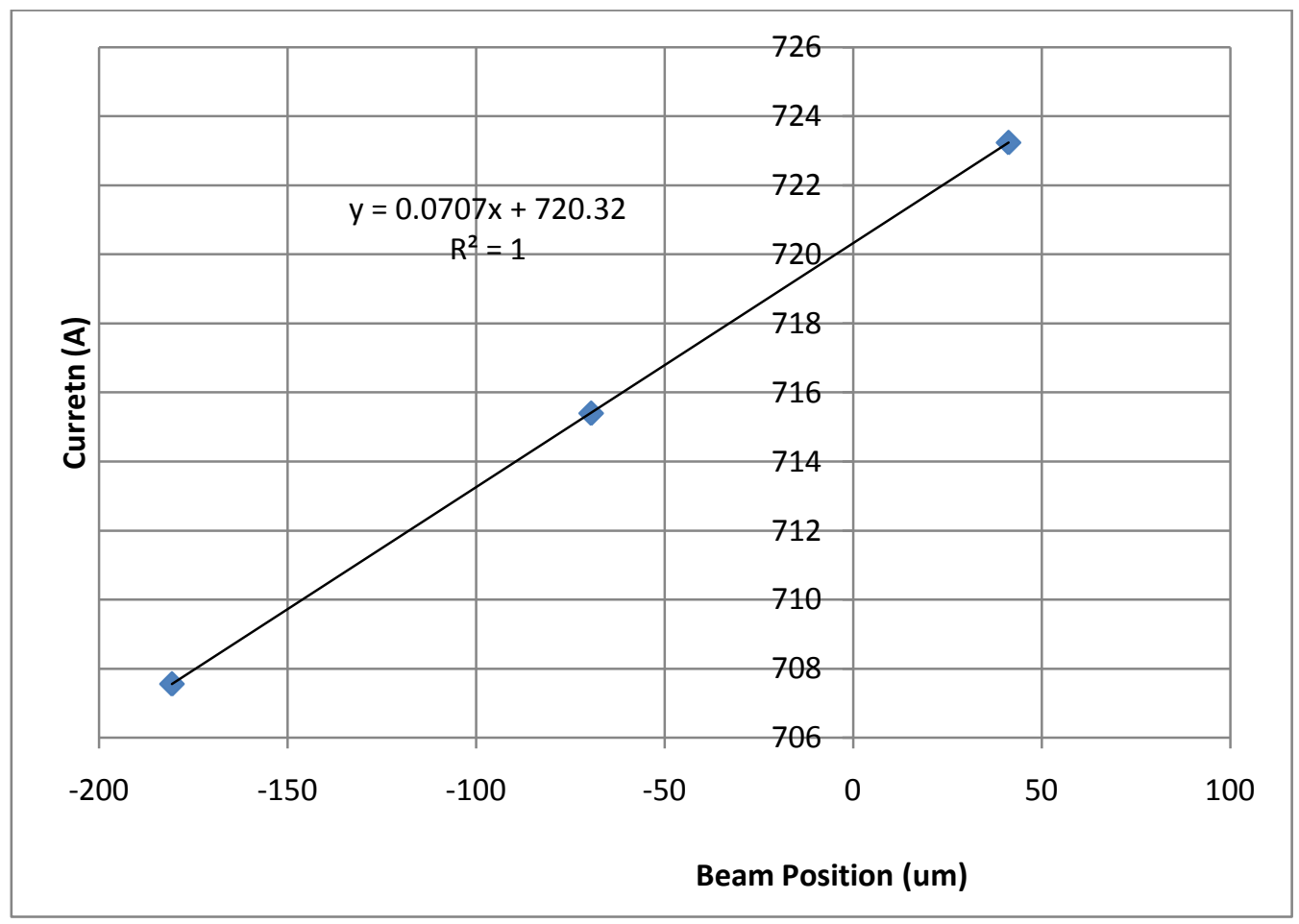

Fig. 13 Power Stability Calculation 
At last, we get the GSB current as the function of beam horizontal position:

$$
Y(A)=0.07 * X(u m)+720
$$

According to our requirements, the beam position fluctuation due to GSB should be less than 10 um, this means power supply stability should less than $0.7 \mathrm{~A}$.

\section{Iron Thickness Estimate for SM Magnet}

In electron lens system, the superconducting main magnet will produce 6 Tesla magnetic fields. If there is no any iron to shield its field, its strong magnetic field may affect the outside instruments or was affected by outside field such as GSB, GS2 and so forth. In this instance, it is difficult to get a uniform field inside of this SM magnet.

To avoid this problem, an iron shield is placed at the outside of superconducting conductor. But the thickness of this iron should be carefully design. It should be compromise between cost and field uniform.

In order to estimate the thickness of iron, we put another straight iron cross over the center of SM magnet. Fig. 14 is the geometry we used for iron thickness estimate. The length of this straight iron line is $100 \mathrm{~cm}$, and the distance between the center of SM and this straight iron line is also $100 \mathrm{~cm}$.

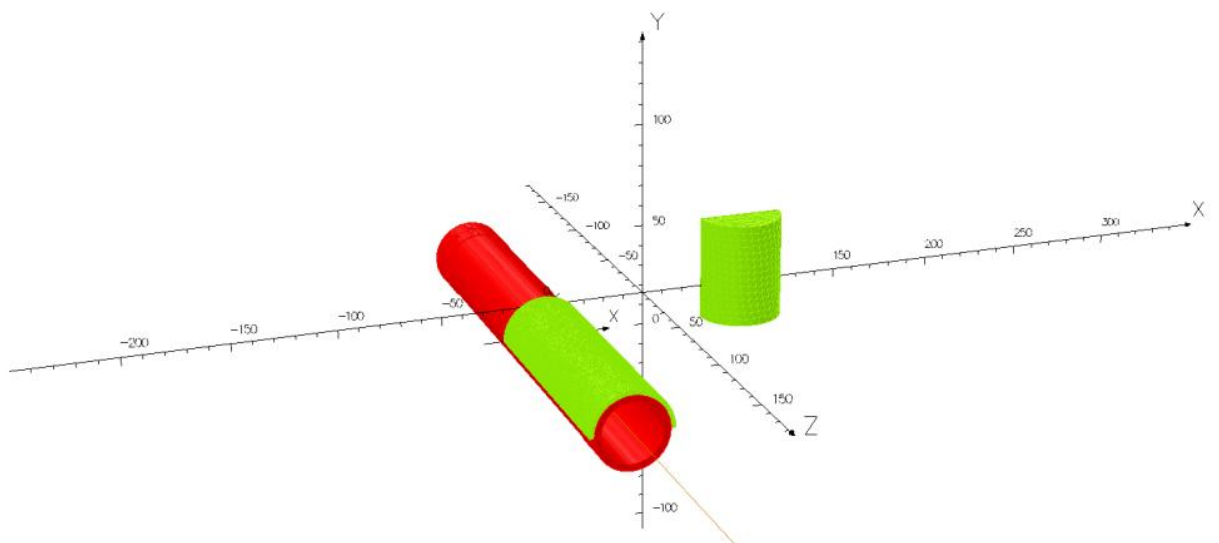

Fig. 14 Simulation Geometry for Iron Thickness Estimate

When we carried our simulation, the diameter of that straight iron and thickness of shield iron were changed. The iron thickness that was used is $2 \mathrm{~cm}, 4 \mathrm{~cm}, 6 \mathrm{~cm}$ and $8 \mathrm{~cm}$ and the diameter of this straight iron line is $40 \mathrm{~cm}$ and $15 \mathrm{~cm}$.

Then the electron beam trajectories were recorded and compared with and without this straight iron line. Their deviations and some other parameters are listed in table 6. 
Table 6 Iron Thickness Simulation

\begin{tabular}{r|r|r}
\hline \hline Thickness of Shield Iron & Deviation with $40 \mathrm{~cm}$ Diameter & Deviation with 15 cm Diameter \\
\hline $2(\mathrm{~cm})$ & $\sim 30 \mathrm{um}$ & $\sim 1.8 \mathrm{um}$ \\
$4(\mathrm{~cm})$ & $\sim 18 \mathrm{um}$ & - \\
$6(\mathrm{~cm})$ & $\sim 17 \mathrm{um}$ & - \\
$8(\mathrm{~cm})$ & $\sim 7 \mathrm{um}$ & - \\
\hline \hline
\end{tabular}

Fig. 15 was the deviation plotted with different thickness of superconducting shield iron. The initial beam position locates at $-120 \mathrm{~cm}$ on $\mathrm{z}$ axis.

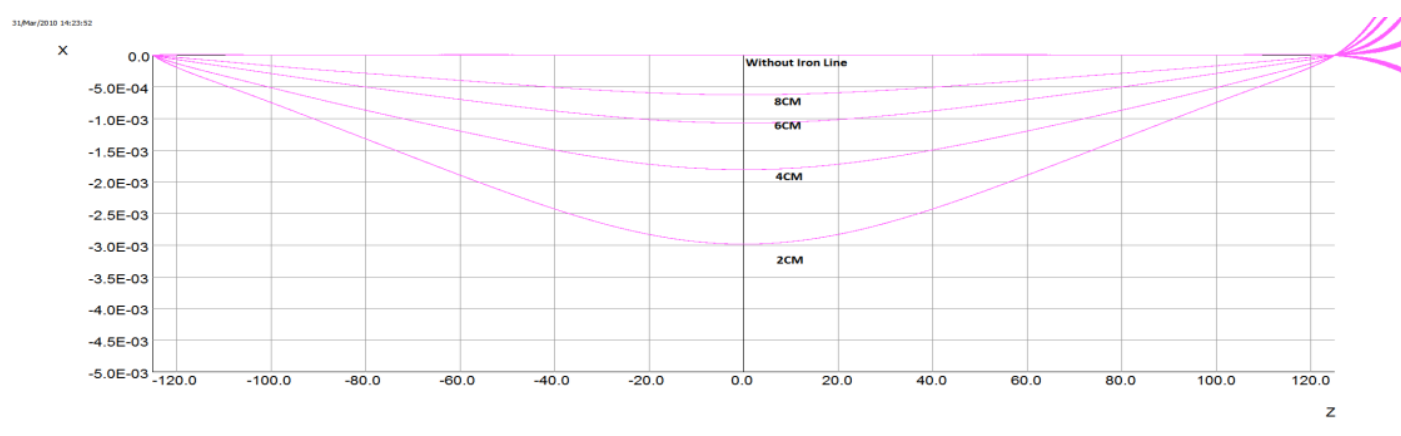

\section{Opera}

Fig. 15 Deviation Simulation with Different Iron Thickness

Fig. 16 is the electron beam trajectories with different thickness ( 9 and $12 \mathrm{~cm}$ ) shield iron. When Fig. 16 was plotted, $10 \mathrm{~cm}$ diameter and $100 \mathrm{~cm}$ length straight iron was placed at $80 \mathrm{~cm}$ from the center of SM magnet.

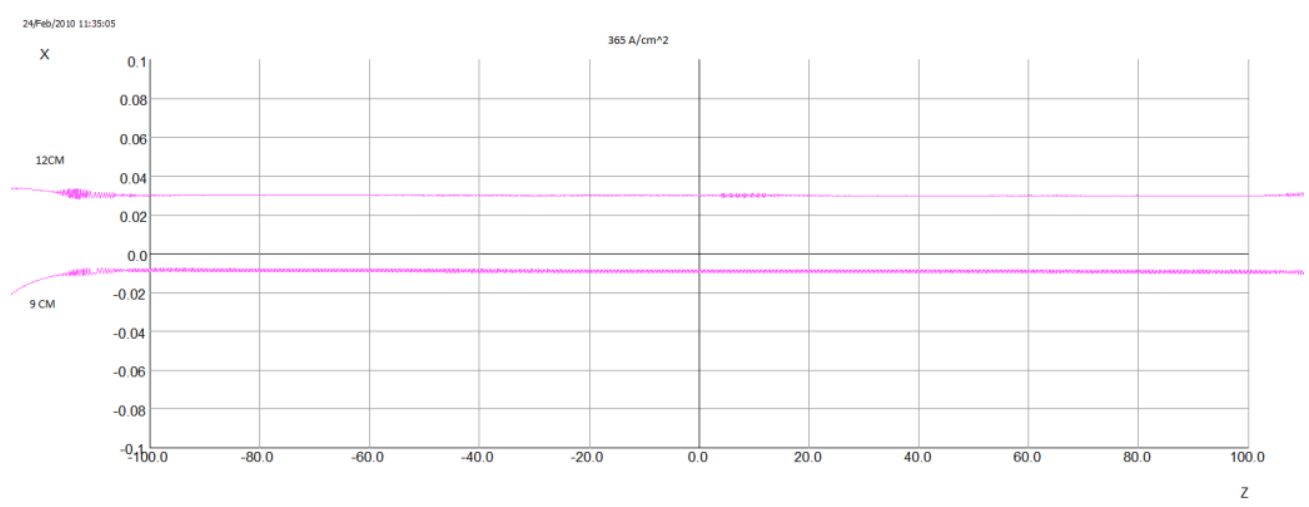

Fig. 16 Beam Trajectories with Different Thickness Iron 
In addition to beam position change, if compared without this straight iron line, the magnetic field at the center of SM magnet also changes 0.25 Gauss and 0.08 Gauss for 9 and $12 \mathrm{~cm}$ thickness respectively.

The thickness of shield iron will be finally designed by superconducting magnet group according to its cost and magnetic field saturation.

\section{Realistic Solenoid Simulation}

Usually, we use idealistic solenoid model in our simulations. The idealistic solenoid has perfect magnetic field flux that is symmetry parallel to the center axis of solenoid. But in fact, the realistic solenoid must have some errors when we make it. These errors will tilt the main magnetic field flux and cause high order magnetic field. Then, this distorted magnetic field will also defect and distort beam trajectories. So, it is also very important to investigate beam behavior after using the realistic solenoids.

After constructing two realistic magnets GS1 and GS2, we put them together with two configurations Case $A$ and Case B, which are shown as Fig. 17. For Case A, GS1 and GS2 have the some leads directions. In Case B, GS1 and GS2 have opposite leads directions.

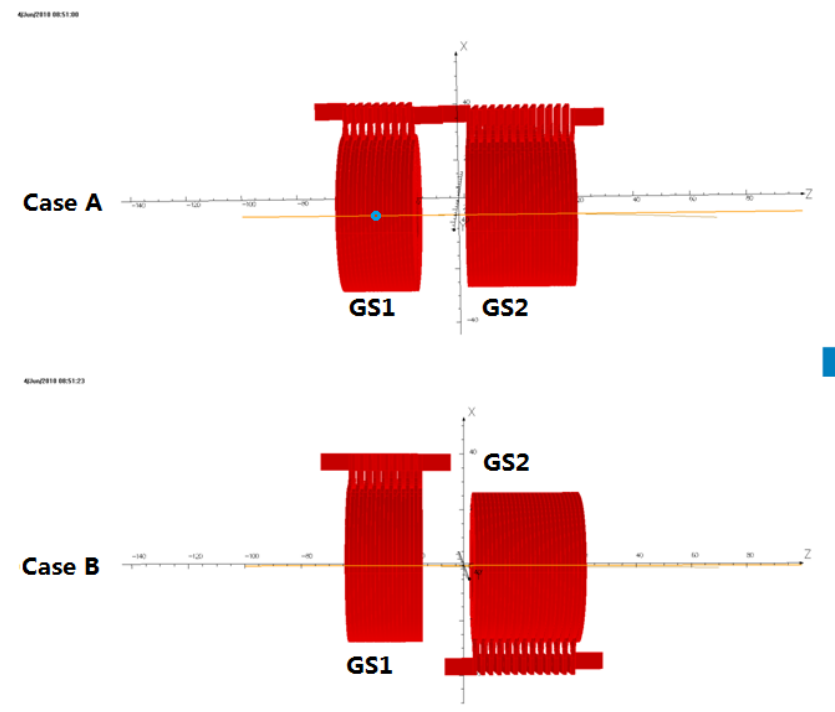

Opera

Fig. 17 Realistic Magnet Simulation with GS1 and GS2

The initial electron beam starts from the center of GS1 and go through GS2 from left to right. Then the beam trajectories are plotted as Fig. 18. 

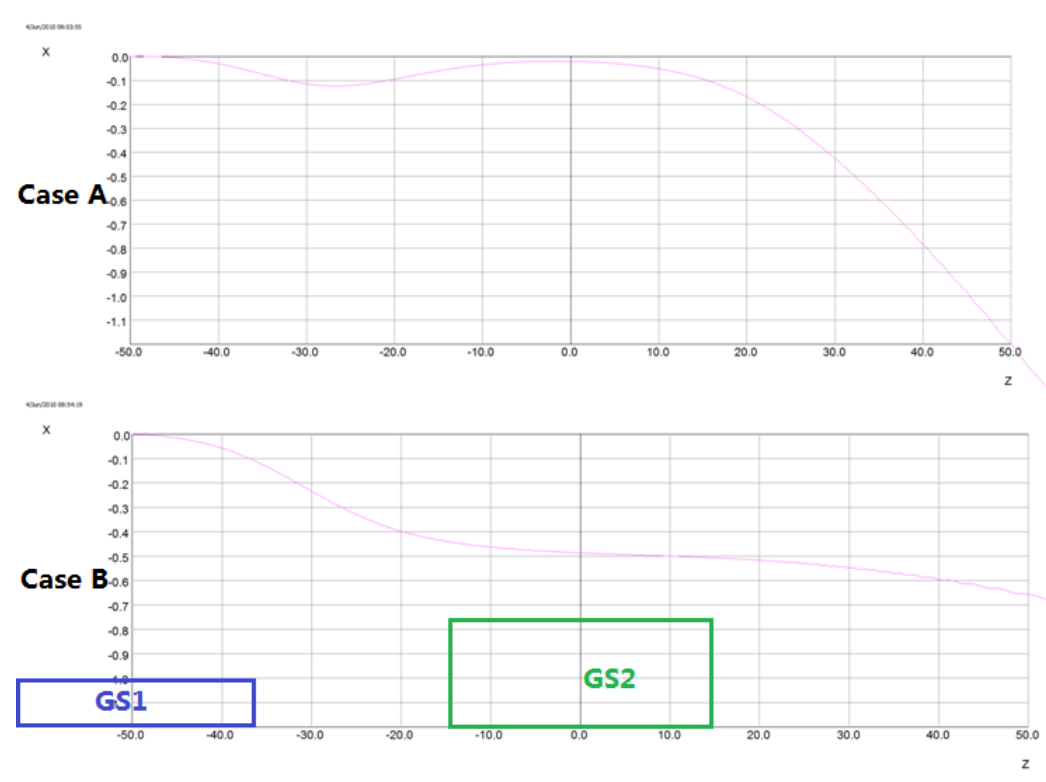

Opera

Fig. 18 Beam Trajectories Calculation by Realistic Magnet

Firstly, from Fig. 20 we can find that electron beam trajectory doesn't go along the center, it is deflected away from the center.

Secondly, we can know that Case A has less position shift inside of GS2 than Case B, but it has larger angle change after passing GS2, this may lead larger position change than Case B. The magnetic field component Bx and By for Case A and B are plotted in Fig. 19.

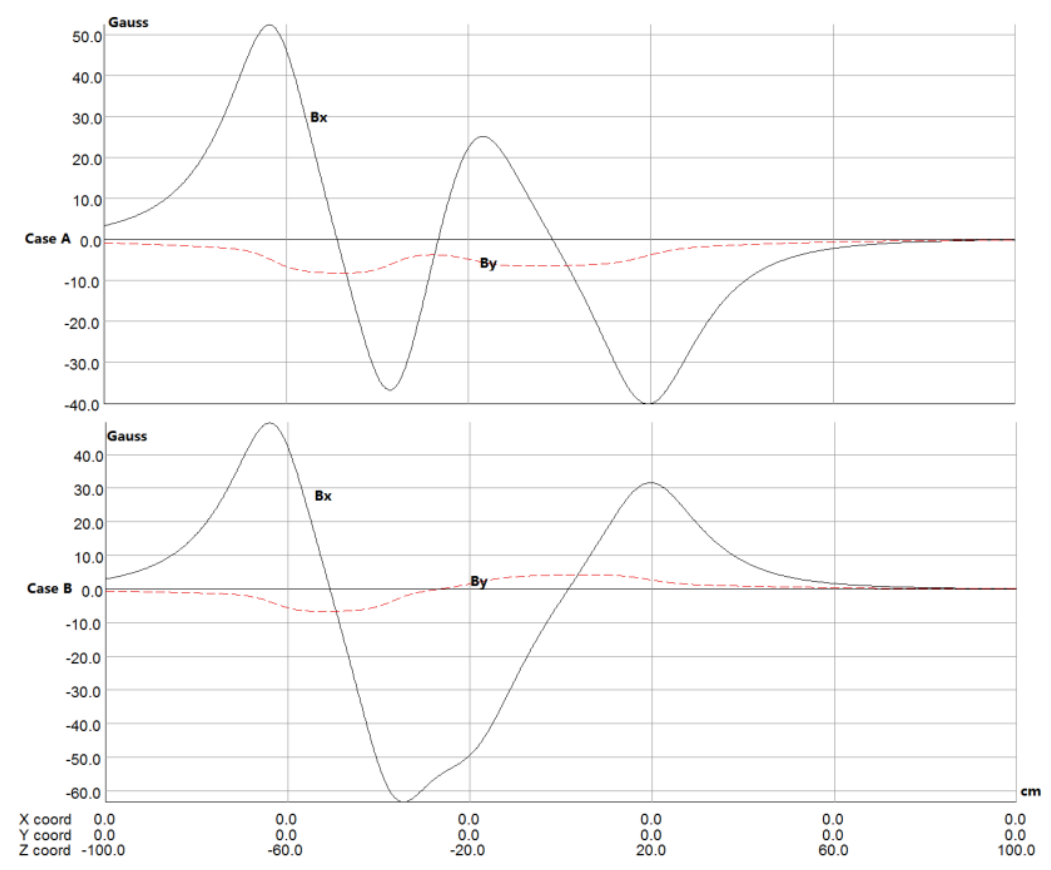




\section{Fig. $19 \mathrm{Bx}$ and By Component of Two Realistic Magnet Configurations}

Furthermore, this beam trajectory change can also be found in our electron beam transport system.

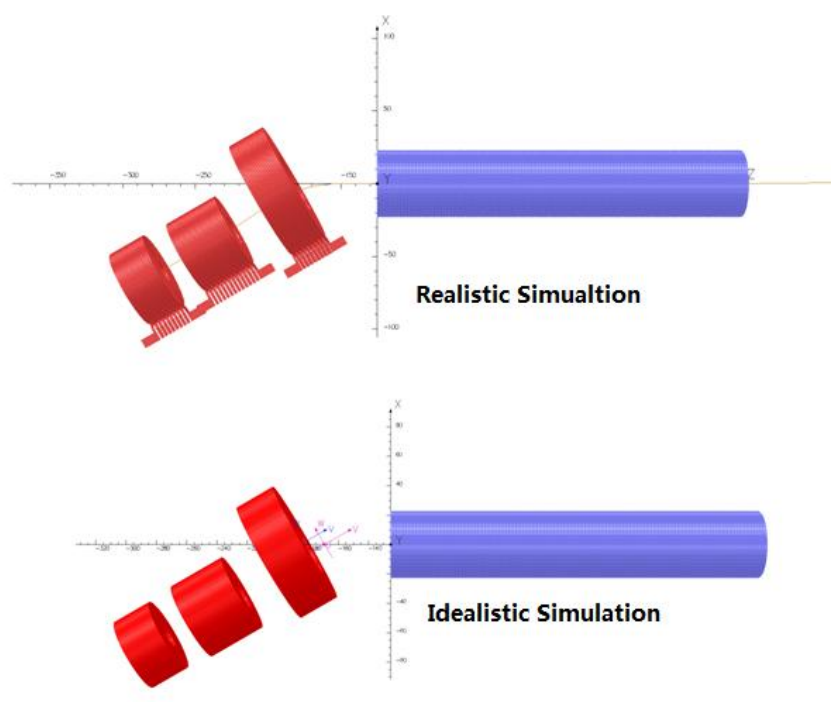

Fig. 20 Realistic Magnet Simulation for Electron Lens

In Fig. 20, after replacing idealistic GS1, GS2 and GSB with realistic magnets, the beam position at the center of SM magnet changes from $(-0.01,-0.01) \mathrm{cm}$ to $(0.08,0.05) \mathrm{cm}$ in horizontal and vertical direction.

\section{Discussion}

Until now, we finished the most important parts of electron lens beam transport system design. For beam position control, the fringe coil in superconducting main magnet may be also used for this purpose.

In this note, we also discussed about some important issues about electron lens project, including two electron lens simulation, realistic magnet simulation and etc. These preparations will make us closer to the success of RHIC electron lens project.

\section{Acknowledgments}

The authors would like to acknowledge D. Gassner and R. Lambiase for their help discussions and useful advices. And the authors must also to thank Walter Shaffer for helpful discussion about realistic solenoid. Of course, the authors wish to thank C. Montag and E. Beebe for their help discussions. 